\title{
Light section measurement to quantify the accuracy loss induced by laser light deflection in an inhomogeneous refractive index field
}

Rüdiger Beermann, Lorenz Quentin, Andreas Pösch, Eduard Reithmeier, Markus Kästner 


\title{
Light Section Measurement to Quantify the Accuracy Loss Induced by Laser Light Deflection in an Inhomogeneous Refractive Index Field
}

\author{
Rüdiger Beermann ${ }^{\mathrm{a}}$, Lorenz Quentin $^{\mathrm{a}}$, Andreas Pösch $^{\mathrm{a}}$, Eduard Reithmeier $^{\mathrm{a}}$, and Markus \\ Kästnera \\ anstitute of Measurement and Automatic Control, Leibniz Universität Hannover, Nienburger \\ Str. 17, 30167 Hannover, Germany
}

\begin{abstract}
In the manufacturing process of Tailored Forming components, the inline inspection of the joining zone directly after each single process step can yield advantages - such as early error detection and real-time process control. Since measuring times need to be synchronized with the production chain, there is no time to cool down the components in between two hot forming processes. On the one hand, the chosen measurement technique needs to be non-tactile due to the heat of the measurement object. On the other hand, the object's areal surface texture needs to be captured rapidly to realize a fast inline inspection. These requirements are only matched by optical 3d measurement systems. Additional challenges arise due to the high temperature of the Tailored Forming components: the ambient air is heated up and the air's temperature increase results in an inhomogeneous refractive index field surrounding the hot workpiece, effecting the light's path emitted by the illumination unit of the optical sensor. We present a simple measurement setup based on the laser light section method to visualize the measurement accuracy loss induced by the convectional heat flow from a hot cylindrical measurement object. To attain a direct validation of the measurement results, the measurements are performed with and with reduced influence of the inhomogeneous refractive index field induced by the hot object.
\end{abstract}

Keywords: 3D measurement, optics, laser light section, refractive index field, light deflection

\section{INTRODUCTION}

Within the Collaborative Research Centre 1153 (CRC) Process Chain for Manufacturing Hybrid High Performance Components by Tailored Forming the 3d geometry of wrought-hot, cylindrical hybrid workpieces and their joining zones have to be characterized directly after the forming process. The immediate inspection and inline condition-monitoring of the geometric features of the joining zones can yield numerous advantages - such as early error detection, reduction of production costs and real-time process control. As the hybrid workpieces can employ temperatures of more than $1000{ }^{\circ} \mathrm{C}$ after each forming process, the chosen metrology method needs to be optical.

Fringe projection or light section methods are commonly used in optical measurement systems. Using the triangulation principle, they rely on the rectilinear projection of light on and reflection of light from the measurement object's surface. Due to the heat transfer of the wrought-hot workpieces to the ambient air, the air will be heated up. The inhomogeneously heated up air results in a density gradient field around the workpiece which again causes a $3 \mathrm{~d}$ inhomogeneous refractive index field, as the density of a medium is directly connected to it's refractive index. A ray of light traversing such a field is deflected towards the more dense air layers, resulting in a deviation from the linear path of the ray. The described effect applies to all kinds of optical triangulation techniques, such as laser light section, fringe pattern projection and stereo-photogrammetry. It decreases the reachable accuracy of the optical sensor system.

To visualize the effect of light deflection on an optical geometry measurement, a simple optical triangulation

Further author information: (Send correspondence to Rüdiger Beermann)

Rüdiger Beermann: E-mail: ruediger.beermann@imr.uni-hannover.de, Telephone: +49511 7625816

Lorenz Quentin: E-mail: lorenz.quentin@imr.uni-hannover.de, Telephone: +49511 7625816

Optical Measurement Systems for Industrial Inspection X, edited by Peter Lehmann, Wolfgang Osten, Armando Albertazzi Gonçalves Jr., Proc. of SPIE Vol. 10329, 103292T · @ 2017 SPIE CCC code: $0277-786 \mathrm{X} / 17 / \$ 18 \cdot$ doi: $10.1117 / 12.2269724$ 


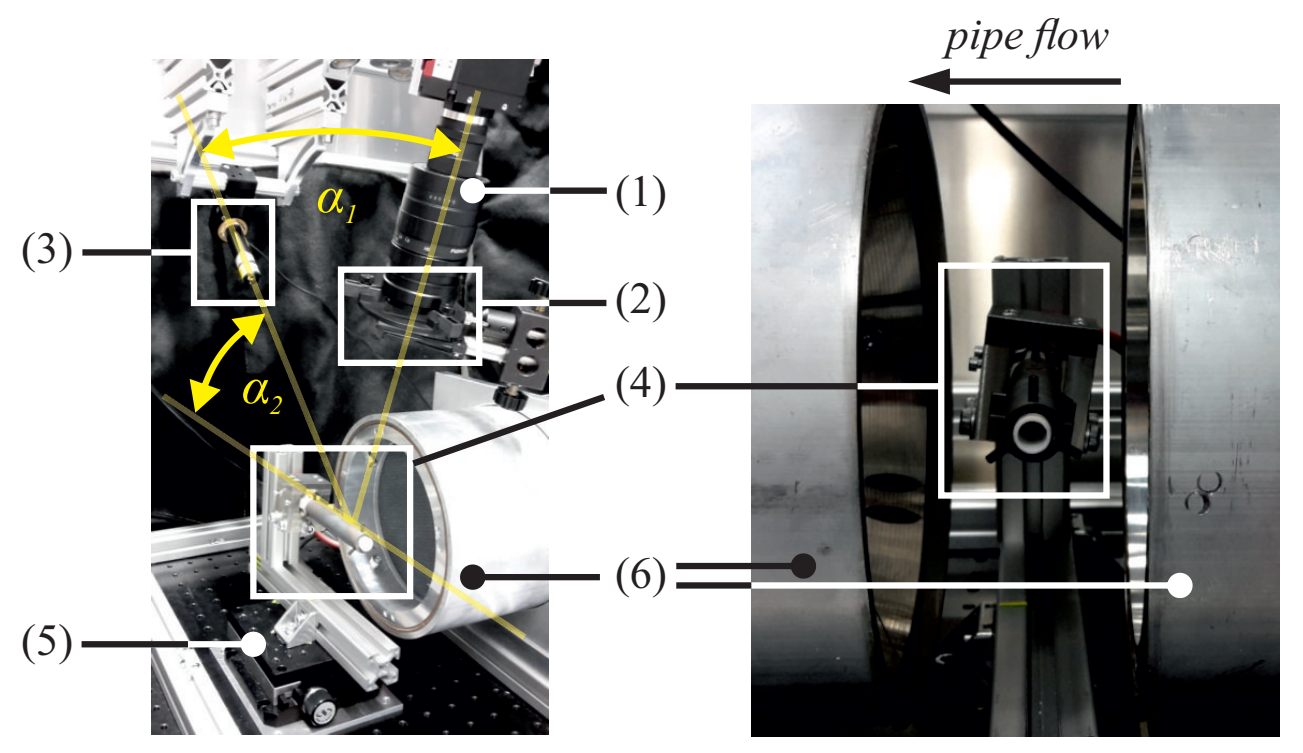

Figure 1: Experimental setup comprising a camera with zoom lens with spacer ring (1), bandpass and NIR filter (2), a laser line generator (3), a cylindrical measurement object with inlaying heating rod (4), a transitional stage (5) and a double pipe system for laminar flow generation (6). The left pipe is missing for visualization purposes in the image on the left-hand side.

sensor based on the laser light section method has been built up at the Institute of Measurement and Automatic Control. To realize $3 \mathrm{~d}$ measurements, the measurement object is moved by a precise translational stage. The measurement object consists of a stainless steel pipe heated up by an inlaying heating rod. Measurements are performed directly through the convective density flow originating from the hot object. To validate the measured geometry data, the hot object's surface is measured with and with reduced effect of the inhomogeneous refractive index field. For this purpose a controlled reduction of the convective heat flow above the measurement object has been achieved by imposing a slow, external laminar flow sideways. The laminar flow is realized by a double pipe system with a flow straightener and two simple computer ventilators.

\section{EXPERIMENTAL SETUP}

\subsection{Triangulation sensor}

The experimental setup is depicted in figure 1. The measurement system comprises a monochromatic camera by $\mathrm{AV}^{1}$ with Gigabit Ethernet Interface (figure 1,(1)). The camera is optimized for extreme environments and fluctuating lighting conditions. It uses a 1"-CMOS-sensor with a resolution of 2048 x 2048 pixels. The pixels are of quadratic shape with $5.4 \mu \mathrm{m}$ lateral length. Using a $75 \mathrm{~mm}$ zoom lens with spacer ring, a field of view of $25 \mathrm{~mm} \times 25 \mathrm{~mm}$ is obtained. To enhance the depth of focus, the aperture of the lens is set to a f-number of 11. The exposure time of the camera is set to $22000 \mu \mathrm{sec}$. In order to achieve the highest possible contrast on the red glowing measurement object, a $20 \mathrm{~mW}$ green laser line generator with cylindrical optic has been integrated in the measurement setup (figure 1,(3)). The triangulation angle $\alpha_{1}$ is $70^{\circ}$, the angle $\alpha_{2}$ between the measurement object's axis and the laser plane is approximately $34^{\circ}$. To separate the measurement signal from incoming radiation induced by self-emission of the hot specimen, a bandpass filter for green light (523 nm) and a NIR filter are used (figure 1,(2)) - similar to the approach of Stöbener et al.. ${ }^{2}$ The camera calibration has been implemented according to Zhang, ${ }^{3}$ but using a planar calibration pattern with circles. The laser plane equation in the camera frame has been obtained by fitting a plane to multiple reprojected laser lines recorded during the calibration process. The basic idea of the laser plane calibration is taken from Abu-Nabah et al.. ${ }^{4}$ To be able to gain multiple sectional views of the measurement object, the object has been attached to a precise transitional stage by Newport ${ }^{5}$ and is moved between consecutive images. To stitch the acquired measurement data correctly, the transitional stage has been calibrated by taking an image of the calibration pattern in every stage position 


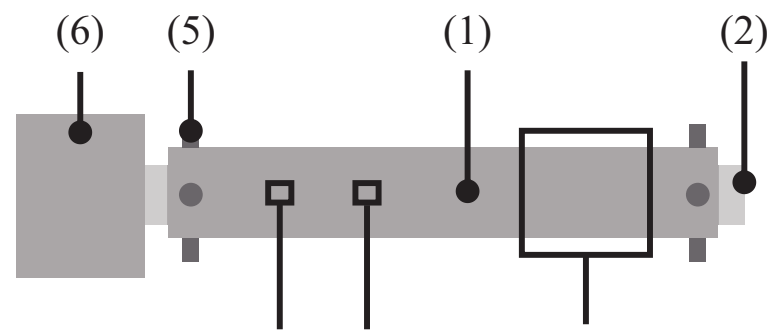

(4.1) (4.2)

(3)

Figure 2: Schematic representation of the measurement object: A cylindrical sandblasted pipe (1) with inlaying heating rod (2), surface measurement zone (3) and temperature measurement locations (4.1) and (4.2). Six screws are arranged radially to fix the cylindrical pipe on the heating rod (5). The heating rod sits in a mounting (6).

that is approached during the measurement task. The surface measurement zone on the measurement object is $12 \mathrm{~mm}$ in axial length (compare to figure 2,(3)). Every $200 \mu \mathrm{m}$ an image of the laser line on the measurement object is to be taken, therefore a single measurement task comprises 61 images or sectional views respectively. A very simple centre of gravity algorithm has been implemented to obtain the centre of the laser lines. Prior to that, the measurement images are undistorted based on the calibration procedure, and a Gaussian filter, a binarization and a morphology operation are applied to the images to improve the robustness of the detection algorithm.

\subsection{Measurement object}

A cylindrical sandblasted pipe (figure 2,(1)) is used as measurement object - made of stainless steel 1.4571 (X6CrNiMoTi17-12-2). It can be heated up to temperatures of about $500{ }^{\circ} \mathrm{C}$ by an inlaying heating rod (figure $2,(2))$. On each end there are three screw-in tap holes to mechanically fix it to the heating rod (figure 2,(5)). The pipe's temperature is measured by a pyrometer, assuming an emission coefficient $\epsilon$ of 0.95 for the used material. The temperature is measured in two different locations (figure 2,(4.1) and (4.2)) - in distance of approximately 30 and $42 \mathrm{~mm}$ to the surface measurement zone (figure 2,(3)).

\subsection{Laminar flow generator}

A double pipe system has been set up to generate a laminar flow. The convective density flow originating from the hot measurement object is meant to be reduced by imposing a slow, external laminar flow sideways (figure $1,(6))$. Thus a validation and interpretation of the measured geometry data can be achieved, as the hot object can be measured from above with and with reduced influence of the inhomogeneous refractive index field that builds up due to heat transfer form the hot object into the ambient air. The right pipe incorporates a flow straightener and two metallic grids with different grid spacing. The air is drawn from the right side into the right pipe by a simple computer ventilator. The left pipe draws the air out of the pipe from the left side - using the same computer ventilator model, but reversely attached to the pipe. The resulting flow direction generated by the pipe system is given in figure 1 on the right-hand side (black arrow). To verify the laminar characteristic of the generated flow, the flow has been visualized with fog fluid placed on curled constantan wire knots. An electric current of about 2 to $3 \mathrm{~A}$ heats up the constantan wire and the fog fluid, giving insight into the stream characteristic (compare to Nitsche et al. ${ }^{6}$ ). The approximate flow velocity has been measured by a simple handheld anemometer $\left(0.8 \mathrm{~m} \mathrm{~s}^{-1}\right)$. To reduce volume shrinkage of the measurement object due to cooling effects by the laminar pipe flow to a minimum, the heating rate of the heating rod is adjusted to control the temperature for the hot measurement object with and without laminar pipe flow.

\section{RESULTS}

\subsection{Measurement conduction and data processing}

Four different measurement scenarios are listed in table 1 (column 1): The cylindrical measurement object has been measured in cold state (state 1), hot state without external laminar pipe flow (state 2), hot state with 
Table 1: Averaged Measurement results based on 5 measurements for each measurement configuration. A cylinder has been fitted to the point clouds using the software GOM Inspect. ${ }^{7}$

\begin{tabular}{|c|c|c|c|c|c|c|}
\hline measurement & $\begin{array}{l}\text { fitted cylinder } \\
\text { radius } r / \mathrm{mm}\end{array}$ & $\begin{array}{c}\text { maximal } \\
\text { deviation to } \\
\text { fitted cylinder } \\
\Delta r_{\max } / \mu \mathrm{m}\end{array}$ & $\begin{array}{c}\text { minimal } \\
\text { deviation to } \\
\text { fitted cylinder } \\
\Delta r_{\min } / \mu \mathrm{m}\end{array}$ & $\begin{array}{c}\text { standard } \\
\text { deviation } \sigma / \mu \mathrm{m}\end{array}$ & $\begin{array}{c}\text { number } \\
\text { of data } \\
\text { points }\end{array}$ & $\begin{array}{l}\text { temperature } T_{1} \\
\text { and } T_{2} /{ }^{\circ} \mathrm{C}\end{array}$ \\
\hline cold object (state 1 ) & $8.325(-)$ & $33.64(-)$ & $-33.42(-)$ & $10.9(-)$ & 50098 & $\begin{array}{c}26.5 \\
-\end{array}$ \\
\hline hot object (state 2 ) & $8.381(+0.67 \%)$ & $58.46(+73.78 \%)$ & $-60.22(+80.19 \%)$ & $18.4(+68.81 \%)$ & 59573 & $\begin{array}{l}434.6 \\
457.8 \\
\end{array}$ \\
\hline $\begin{array}{c}\text { hot object with } \\
\text { laminar pipe flow } \\
\text { (state } 3)\end{array}$ & $8.446(+1.45 \%)$ & $33.86(+0.65 \%)$ & $-33.20(-0.66 \%)$ & $10.7(-1.83 \%)$ & 60095 & $\begin{array}{l}423.1 \\
454.1\end{array}$ \\
\hline $\begin{array}{l}\text { hot object with } \\
\text { pulsed laminar pipe } \\
\text { flow (state } 4 \text { ) }\end{array}$ & $8.424(+1.19 \%)$ & $44.00(+30.80 \%)$ & $-43.64(+30.58 \%)$ & $13.6(+24.77 \%)$ & 60291 & $\begin{array}{l}427.32 \\
458.68\end{array}$ \\
\hline
\end{tabular}

laminar pipe flow (state 3) and in hot state with pulsed laminar pipe flow (state 4). The pulsed laminar pipe flow has been achieved by switching the pipes' computer ventilators on and off every 20 s. For this, the measurement started with powered ventilators for the duration of $20 \mathrm{~s}$, then the ventilators have been switched off for the next $20 \mathrm{~s}$ and so forth.

For every scenario five complete measurements have been conducted, leading to an amount of $5 \mathrm{x} 61$-sectional views in each scenario. As a single measurement procedure takes approximately $135 \mathrm{~s}$, the temperature has been monitored at the beginning of every measurement.

Based on the five measurements for each scenario, specific averaged values have been derived and are given in table 1. A cylinder has been fitted to the triangulated data points using the Software GOM Inspect. ${ }^{7}$ The measured, averaged fitted cylinder radius $r$ is given in column 2. Statistical outliers have been omitted in the Gaussian cylinder fitting process by defining a $\pm 3 \sigma$-boundary range. Furthermore the averaged maximal and minimal deviation from the fitted cylinder $\Delta r_{\max }$ and $\Delta r_{\min }$, the averaged standard deviation $\sigma$, the averaged number of data points and the averaged temperature $\left(T_{1}\right.$ and $T_{2}$ for the locations (4.1) and (4.2) in figure 2) during the measurement procedure is given in table 1. The results of the cold measurement serve as reference for the hot measurement results. Therefore the value changes are additionally given on a percentage basis in brackets behind the absolute values in comparison to the cold reference measurement.

\subsection{Discussion}

The averaged fitted cylinder radius increases with rising temperature (see table 1, column 2). Interestingly, the averaged radius of the hot object with laminar pipe flow (state 3) increases more intensely than the radius of the hot object without laminar flow (state 2 ) - although the temperatures $T_{1}$ and $T_{2}$ don't change significantly. Furthermore, the averaged minimal and maximal deviations $\Delta r_{\max }$ and $\Delta r_{\min }$ are smaller for state 3 compared to state 2. The same applies for the averaged standard deviation $\sigma$.

The results in table 1 are averaged values for five measurements, therefore exemplary the triangulated laser lines (see figure 3) and the deviation from the fitted cylinder (see figure 4) are depicted for the first measurement in a certain measurement scenario.

First of all, the parallelism of the laser lines in figure 3 for the cold (a) and the hot measurement object with laminar flow (c) is noticeable ${ }^{\dagger}$. The parallelism of the laser lines results in similar maximal and minimal deviation values for the fitted cylinder (compare to figure 4 (a) and (c)).

A different laser line alignment can be identified in figure 3 for the measurement configuration without external flow (b) and pulsed laminar flow (d). For the mere hot object, the distance between the laser lines is not as equidistant as in the cold or laminar scenario, but seems to be rather arbitrarily distributed. This arbitrariness results in a contour plot that shows a surface structure with irregularities and uneven sections (compare to figure

\footnotetext{
${ }^{\dagger}$ For more detail a zoomed section is depicted in black frames in figure 3.
} 


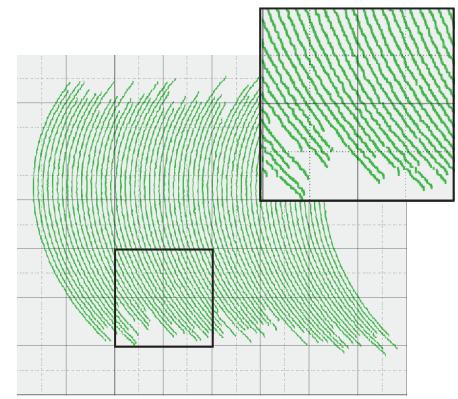

(a) Cold object

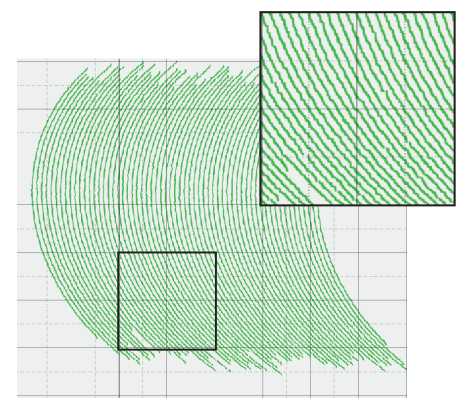

(c) Hot object with laminar pipe flow

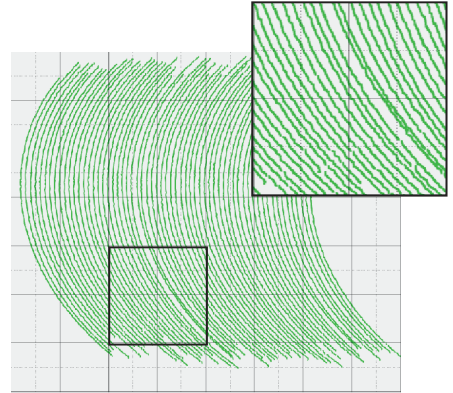

(b) Hot object

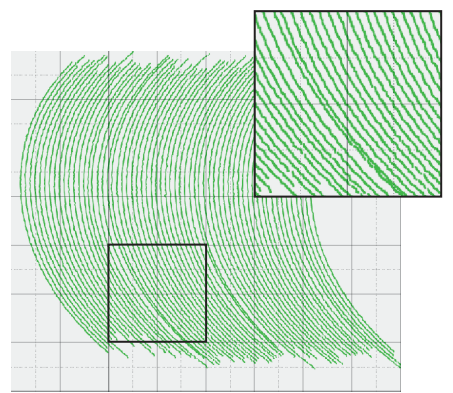

(d) Hot object with pulsed laminar pipe flow

Figure 3: Triangulated laser lines for different measurement scenarios ((a) to (d)). Exemplary only the first measurement of a measurement series is depicted.

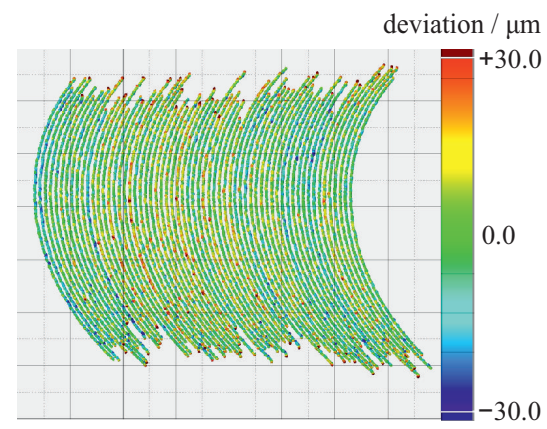

(a) Cold object

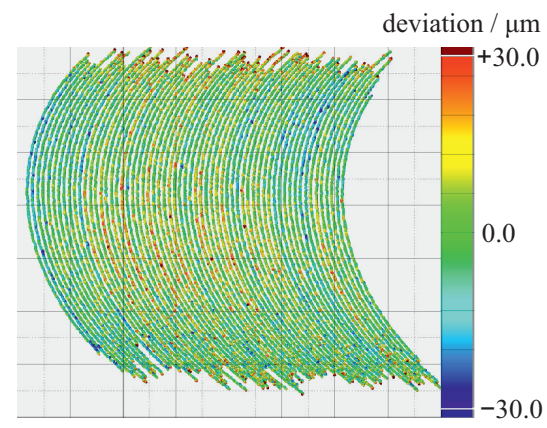

(c) Hot object with laminar pipe flow

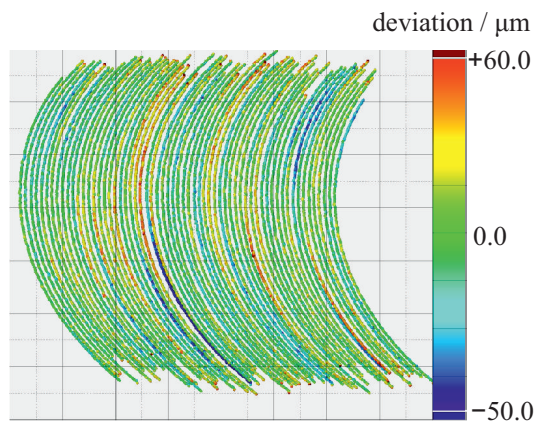

(b) Hot object

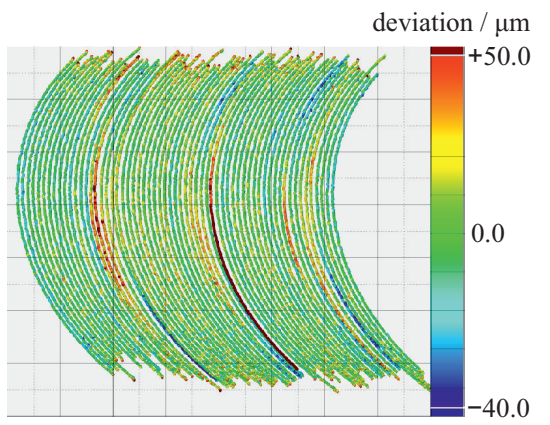

(d) Hot object with pulsed laminar pipe flow

Figure 4: Deviation of measurement points from fitted cylinder for different measurement scenarios ((a) to (d)). Exemplary only the first measurement of a measurement series is depicted. 


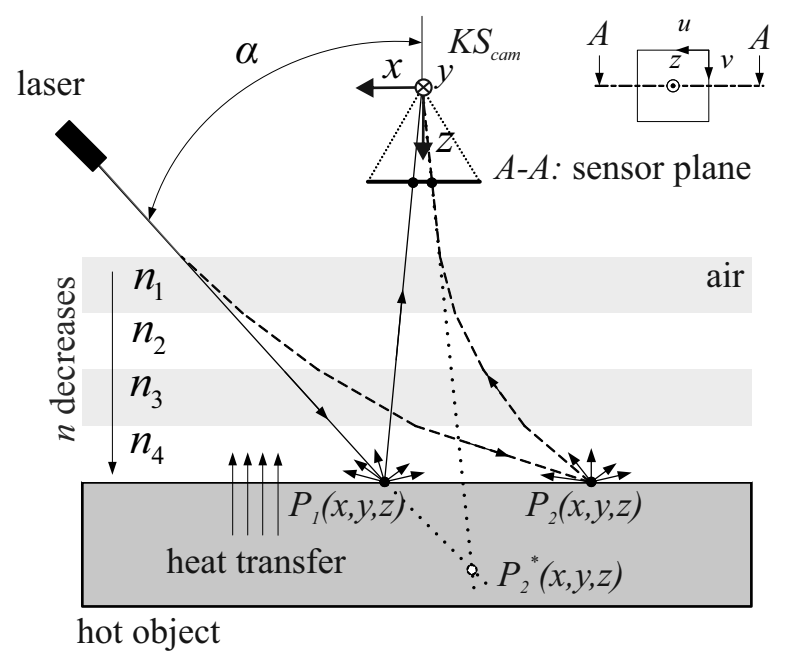

Figure 5: Simplified 2d outline of the laser light deflection due to an inhomogeneous refractive index field induced by an hot object. ${ }^{8}$

4 (b)). The configuration with pulsed laminar flow reveals the effect of the external pipe flow - the deviation seems to rise for regions with ventilators turned off, whereas powered ventilators result in evenly spaced laser lines and a more regular surface area.

\subsection{Conclusions}

In the presented paper the deflection effect on the laser line of an triangulation sensor due to an inhomogeneous refractive index field has been examined. Although the absolute values for the different measurement scenarios given in table 1 are only valid for the specific measurement setup in figure 1, a general conclusion can be derived: The accuracy of an optical sensor is verifiable effected by refractive index variations induced by a hot measurement object - at least for an object's temperature of roughly $450{ }^{\circ} \mathrm{C}$ or more and provided that the measurement is performed through the convective density flow. If the measurement results of the scenario with hot object and external laminar pipe flow are assumed to represent the hot object's surface more accurately than without laminar flow, the accuracy loss due to the inhomogeneous refractive index field can be derived by analysing the deviation from the fitted cylinders in figure 4 for the cases (b) and (c). The associated colour legends give the deviations based on a $3 \sigma$ range - the extremal values increase from $\pm 30 \mu \mathrm{m}$ to +60 and $-50 \mu \mathrm{m}$ respectively. Furthermore, the hot object's measured radius has been determined to be smaller than the radius in the scenario with laminar pipe flow.

To fully understand the described effect, a simplified $2 \mathrm{~d}$ outline of the triangulation error due to light deflection is depicted in figure $5 \ddagger$ The camera sensor (2d sensor plane (A-A)) is arranged directly above the hot surface. The refractive index is assumed to decrease in discrete layers. The nearer the air layers are located to the hot object's surface, the smaller is the refractive index. Assuming an homogeneous refractive index field, the laserspot is reconstructed via triangulation in location $P_{1}(x, y, z)$ (figure 5 , solid line). In the scenario with laser light deflection (dashed line, $P_{2}(x, y, z)$ ), the triangulated surface point is reconstructed in the wrong location (dotted line, $\left.P_{2}^{*}(x, y, z)\right)$. During the calibration procedure of the sensor the refractive index was assumed to be constant - therefore the curvature of the laser light path has not been modelled.

Transferring these conclusions to the experimental setup leads to the following: As the inhomogeneous refractive index field changes dynamically, the laser line is "flaring" on the hot object. The deflection effect differs for different laser line pixel in the camera image. This fluctuation leads to an increasing and decreasing triangulation error, as the detected laser line centre is changing not only in dependency of the object's height, but also in dependency of the air's temperature.

\footnotetext{
${ }^{\ddagger}$ The figure is taken from Beermann et al.. ${ }^{8}$ The journal paper is accepted and will be published in 2017.
} 


\section{FUTURE WORK}

The results in this paper give a first insight into the challenges that arise, if a hot object's areal surface is to be measured optically. Unfortunately, it is not easy to adjust the calibration procedure of an optical sensor to the dynamically changing refractive index field of a hot object. One approach to reduce the effect of the inhomogeneous refractive index field on the accuracy is a measurement from the bottom, omitting surface areas that are highly influenced by the convective density flow. This approach is not an option, if the $3 \mathrm{~d}$ surface data has to be obtained from all sides without moving the measurement object. Therefore, first experiments have been conducted at the Institute of Measurement and Automatic Control to realize measurements in rough vacuum, thereby reducing the refractive index variations in air significantly. Although this approach is rather complex as the vacuum chamber needs to be equipped with an automatic feeding system for the hot components, the first measurement results using a fringe projection system are promising.

\section{ACKNOWLEDGMENTS}

The results presented in this paper were obtained within the Collaborative Research Centre 1153 Process Chain for Manufacturing Hybrid High Performance Components by Tailored Forming in the subproject C5 Multiscale Geometry Inspection of Joining Zones. The authors would like to thank the German Research Foundation (DFG) for the financial and organisational support of this project.

\section{REFERENCES}

[1] AlliedVision, "Prosilica GT 2050." https://www.alliedvision.com/en/products/cameras/detail/ Prosilica\%20GT/2050.html (2017). (Accessed: 06 April 2017).

[2] Stöbener, D., Dijkman, M., Kruse, D., Surm, H., Keßler, O., Mayr, P., and Goch, G., "Distance measurements with laser-triangulation in hot environments," integration $\mathbf{3}(2), 5$ (2003).

[3] Zhang, Z., "A flexible new technique for camera calibration," IEEE Transactions on Pattern Analysis and Machine Intelligence 22(11), 1330-1334 (2000).

[4] Abu-Nabah, B. A., ElSoussi, A. O., and Al Alami, A. E. K., "Simple laser vision sensor calibration for surface profiling applications," Optics and Lasers in Engineering 84, 51-61 (2016).

[5] Newport, "Newport M-VP-25XA." https://www.newport.com/p/M-VP-25XA (2017). (Accessed: 06 April 2017).

[6] Nitsche, W. and Brunn, A., [Strömungsmesstechnik], Springer-Verlag (2006).

[7] GOM, "GOM Inspect 2016." http://www.gom-inspect.com/de/index.php (2017). (Accessed: 06 April 2017).

[8] Beermann, R., Quentin, L., Pösch, A., Reithmeier, E., and Kästner, M., "Background oriented schlieren measurement of the refractive index field of air induced by a hot, cylindrical measurement object," Applied Optics (2017). 